\title{
Prevalence of Unstable Attractors in Networks of Pulse-Coupled Oscillators
}

\author{
Marc Timme, Fred Wolf, and Theo Geisel \\ Max-Planck-Institut für Strömungsforschung and Fakultät für Physik, Universität Göttingen, 37073 Göttingen, Germany
}

(Received 22 February 2002; published 23 September 2002)

\begin{abstract}
We present and analyze the first example of a dynamical system that naturally exhibits attracting periodic orbits that are unstable. These unstable attractors occur in networks of pulse-coupled oscillators, and become prevalent with increasing network size for a wide range of parameters. They are enclosed by basins of attraction of other attractors but are remote from their own basin volume such that arbitrarily small noise leads to a switching among attractors.
\end{abstract}

DOI: $10.1103 /$ PhysRevLett.89.154105

PACS numbers: 05.45.-a, 87.10.+e, 89.75.-k

As attractors determine the long-term behavior of dissipative dynamical systems, the concept of attractors is central to the analysis of many natural systems as well as to the design of artificial systems. For instance, the computational capabilities of neural networks are controlled by the attractors of their collective dynamics. Consequently, the nature and design of attractors in such systems constitute a focus of current research [1-3]. In general, the state space of a nonlinear dynamical system is partitioned into various basins of attraction from which states evolve towards the respective attractors. Since states that are slightly perturbed from an attractor often stay confined to its vicinity and eventually return to the attractor, attractors are commonly considered to be stable [4].

In the present Letter, we show that unstable attractors exist and arise naturally as a collective phenomenon in networks of pulse-coupled oscillators [1,2], which were introduced to model, e.g., synchronization in spiking neural networks and the dynamics of other natural systems as diverse as cardiac pacemaker cells, populations of flashing fireflies, and earthquakes (cf. [1-3,5]). We identify an analytically tractable network exhibiting unstable attractors. For this network we demonstrate the existence of attractors that are linearly unstable and are thus separated from the volume of their own basins of attraction. Such attracting yet unstable states are consistent with a definition of attractors introduced by Milnor, which neither presumes nor implies stability [6]. In some other systems such Milnor attractors might not be uncommon if they are strange attractors that display irregular dynamics [7]. More generally, however, attractors that are not stable seem to be special cases that have to be constructed artificially by precisely tuning parameters. Contrary to this intuition, we report here that unstable attractors with regular, periodic dynamics are typical in large networks and persist even if the physical parameters are varied substantially.

We argue that dynamical consequences of unstable attractors may persist in a general class of systems of pulse-coupled units. Such consequences include an ongoing switching among unstable attractors in the presence of noise. In systems where the convergence towards an attractor has a functional role, such as the solution of a computational task by a neural network [8], switching induces a high degree of flexibility that provides the system with a unique advantage compared to multistable systems: It will be hard to leave a stable attractor after convergence, e.g., the completion of a task. With an unstable attractor, however, a small perturbation is sufficient to leave the attractor and to switch towards another one.

We consider a homogeneous network of $N$ all-to-all pulse-coupled oscillators with delayed interactions. A phase variable $\phi_{i}(t) \in[0,1]$ specifies the state of each oscillator $i$ at time $t$. Its free dynamics is given by

$$
d \phi_{i} / d t=1 \text {. }
$$

Whenever oscillator $i$ reaches a threshold, $\phi_{i}(t)=1$, the phase is reset to zero, $\phi_{i}\left(t^{+}\right)=0$, and a pulse is sent to all other oscillators $j \neq i$, which receive this signal after a delay time $\tau$. Depending on whether the input $\hat{\varepsilon}$ is subthreshold or suprathreshold, this induces a phase jump according to

$$
\phi_{j}\left((t+\tau)^{+}\right)=\min \left\{U^{-1}\left(U\left(\phi_{j}(t+\tau)\right)+\hat{\varepsilon}\right), 1\right\}
$$

which depends on the phase $\phi_{j}(t+\tau)$ of the receiving oscillator and the effective excitatory coupling $\hat{\varepsilon}=$ $\varepsilon /(N-1)>0$. The function $U(\phi)$ is twice continuously differentiable, monotonously increasing, $U^{\prime}>0$, concave (down), $U^{\prime \prime}<0$, and normalized such that $U(0)=0$, $U(1)=1$. For many models of biological systems $U(\phi)$ represents a "potential" of an oscillator at phase $\phi$. For a more detailed discussion of the model see Refs. [1,2].

For such pulse-coupled systems, periodic orbits with groups of synchronized units constitute relevant attractors [1-3,5]. For instance, the network described above possesses a single global attractor in which all oscillators are synchronized with zero phase lag if the interactions are instantaneous $(\tau=0)$ [1]. Here we consider the case of delayed interactions $(\tau>0)$ where multiple different cluster-state attractors with several synchronized groups of oscillators (clusters) coexist [2]. Such attractors are period-one orbits with all oscillators in the clusters reaching threshold and sending out pulses exactly once 

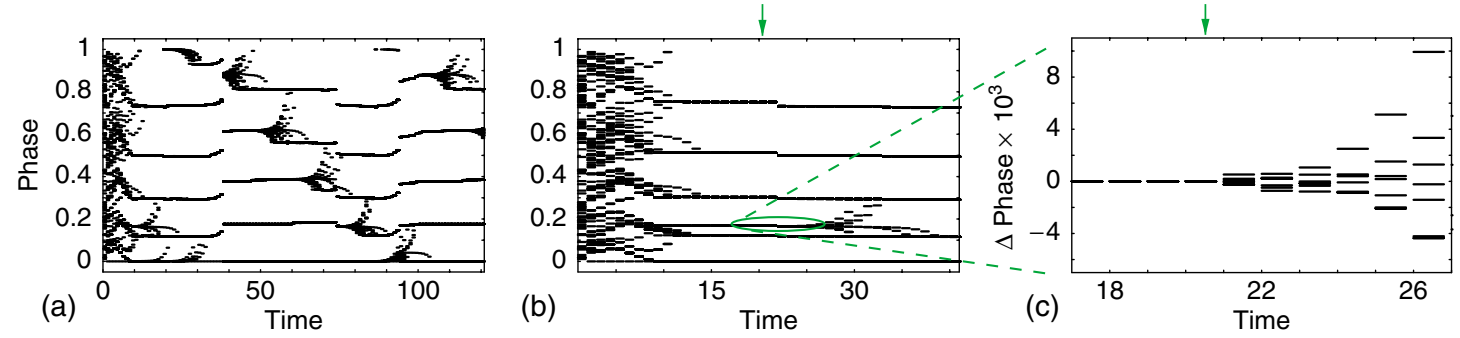

FIG. 1 (color). Phase dynamics of a large network $(N=100, \varepsilon=0.2, \tau=0.15)$. Phases of all oscillators are plotted whenever a reference oscillator has been reset. (a) Dynamics with noise $\left(\eta=10^{-3}\right)$, (b) deterministic dynamics in response to a single phase perturbation (arrow, $\sigma=10^{-3}$ ) — note that the system switches from a six-cluster to a five-cluster state-and (c) phase differences from the average phase of one cluster in response to the perturbation.

during each period. We find [cf. Fig. 1(a)] that, although the system converges towards a periodic orbit from random initial conditions, weak noise is often sufficient to drive the system away from that attractor such that successive switching towards different attractors occurs. This alternating synchronization and desynchronization might be due to stable attractors located close to the boundaries of their basins of attraction, such that the noise drives the state of the system into a neighboring basin. In an otherwise noiseless system we tested this possibility by applying instantaneous perturbations of gradually decreasing strengths [down to $\sigma=10^{-8}$; cf. Figs. 1(b) and 1(c)]. As we did not find a strength for which any of the perturbed states returned to the attractor, we hypothesized that the persistent switching dynamics [Fig. 1(a)] is due to attractors that are unstable.

In order to verify this hypothesis directly, we analyze a small network of $N=6$ oscillators for which instantaneous perturbations lead to a similar switching among attractors. At given parameters [9] this network exhibits a set of period-one orbits that are related by a permutation of phases in such a way that the system may switch among them [Fig. 2(a), states on the periodic orbits marked in red, yellow, blue]. Because of their permutation equivalence these orbits have identical stability properties. The state of the network at time $t$ is specified by $\boldsymbol{\phi}(t)=\left(\phi_{1}(t), \ldots, \phi_{6}(t)\right)^{\top}$, such that the orbit marked in yellow in Fig. 2(a) is defined by the initial condition [10]

$$
\boldsymbol{\phi}(0)=(0,0, A, A, B, C)^{\top} .
$$

Here the origin of time was chosen such that oscillators 1 and 2 have just sent a signal and have been reset. Moreover, at $t=0$ only these two signals (and no others) have been sent but not yet received. The numerical values for the particular parameters considered, $A \approx 0.176, B \approx$ 0.499, $C \approx 0.747$, can be identified in Fig. 2(a) (orbit marked in yellow). This orbit indeed is periodic, $\boldsymbol{\phi}(T)=$ $\boldsymbol{\phi}(0)$, such that after the period $T$ each oscillator has reached threshold, has sent a signal, and has been reset exactly once (for details see [11]).

To perform a stability analysis, we define a return map by choosing oscillator $i=1$ as a reference: Let $\phi_{n, i}:=$ $\phi_{i}\left(t_{n}\right)$ be the perturbed phases of the oscillators $i$ at times $t_{n}>0, n \in \mathbb{N}$, just after the resets of oscillator 1 , $\phi_{1}\left(t_{n}\right) \equiv 0$. Thus the five-dimensional vector $\boldsymbol{\delta}_{\boldsymbol{n}}=\boldsymbol{\phi}_{\boldsymbol{n}}-$ $\left(0, A, A, B, C^{\prime}\right)^{\top}$ (see [12]) defines the perturbations $\delta_{n, i}$ for $i \in\{2, \ldots, 6\}$ where we choose $0<\delta_{n, 2}$ and $\delta_{n, 3}<$ $\delta_{n, 4}$. Following the dynamics, the fivedimensional return map is given by [13]

$$
\boldsymbol{\delta}_{n+1}=\boldsymbol{F}\left(\boldsymbol{\delta}_{n}\right) .
$$

The linearized dynamics of a slightly perturbed state

(a)
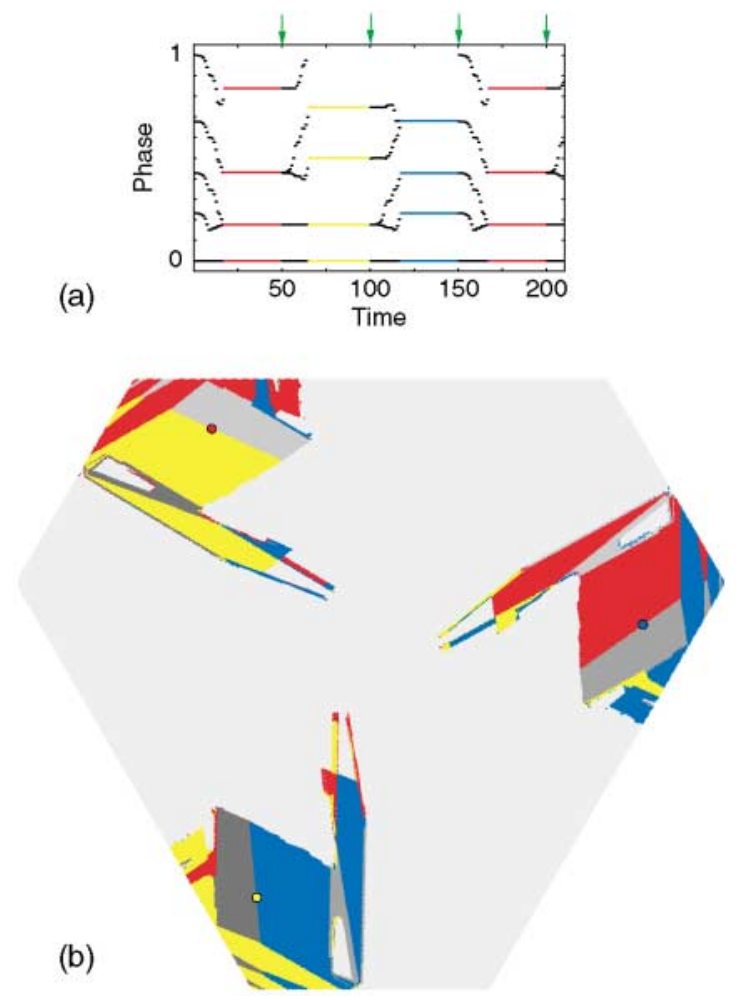

FIG. 2 (color). Small network $(N=6, \varepsilon=0.2, \tau=0.15)$ : (a) Noise-free phase dynamics in response to single perturbations (arrows). (b) Basin structure in a two-dimensional planar section through six-dimensional state space. Small red, yellow, and blue disks represent points on the attractors color-marked in (a). Their basins of attraction are marked in the same colors. Medium gray areas are basins of permutation-related attractors, and the lightest gray marks the union of the basins of all other attractors. 
with split-up clusters is described by the Jacobian matrix $M=\partial \boldsymbol{F}(\boldsymbol{\delta}) /\left.\partial \boldsymbol{\delta}\right|_{\boldsymbol{\delta}=\mathbf{0}}$. It has four zero eigenvalues

$$
\lambda_{i}=0 \quad \text { for } i \in\{1,2,3,4\},
$$

such that a six-dimensional state-space volume accessed by the perturbation is contracted onto a two-dimensional manifold. This reflects the fact that suprathreshold input received simultaneously by two or more oscillators leads to a simultaneous reset and thus a synchronization of these oscillators independent of their precise phases. If a single oscillator is reset by a suprathreshold input signal, it exhibits a precise lag in firing time $\Delta t=\tau$ compared to the oscillator that has sent this signal. In contradistinction, the concavity of $U$ implies that simultaneous subthreshold input to two or more oscillators leads to an increase of their phase differences, i.e., a desynchronization of oscillators with similar phases. For the orbits considered here, this is reflected by the only nonzero eigenvalue

$$
\lambda_{5}=\frac{\left(2 U^{\prime}\left(c_{0}\right)-U^{\prime}\left(a_{1}\right)\right) U^{\prime}\left(c_{1}\right) U^{\prime}\left(c_{2}\right) U^{\prime}\left(c_{3}\right)}{U^{\prime}\left(a_{1}\right) U^{\prime}\left(a_{2}\right) U^{\prime}\left(a_{3}\right) U^{\prime}\left(a_{4}\right)}>1
$$

where $c_{i}=\tau+a_{i}$ for all $i$ (cf. [10]). Because $c_{i}>a_{i}>$ $c_{i-1}$ for all $i$ and $U^{\prime}>0, U^{\prime \prime}<0$, this eigenvalue is larger than one; i.e., the periodic orbit is linearly unstable. If there is no homoclinic connection, this implies that such an attractor is not surrounded by a positive volume of its own basin of attraction, but is located at a distance from it: Thus, every random perturbation to such an attractor state - no matter how small-leads to a switching towards a different attractor. Furthermore, this periodic orbit indeed is an attractor: Right after the perturbation off a periodic orbit [e.g., the one marked in red in Fig. 2(a), which is permutation equivalent to the yellow one] the state of the system is mapped onto a twodimensional manifold, resynchronizing one cluster. The state then evolves towards a neighborhood of another attractor (here, the yellow one) in a lower dimensional effective state space without further dimensional reduction. Here, forming the second cluster, suprathreshold input leads to the last dimensional reduction while the state is mapped directly onto the periodic orbit.

In general, a periodic orbit is unstable, if after a random perturbation into its vicinity, one or more clusters are not resynchronized by simultaneous suprathreshold input but desynchronize due to simultaneous subthreshold input. An unstable attractor results if these clusters are formed through synchronization in a region of state space that is separated from the periodic orbit towards which the state then converges. Roughly, unstable attractors can be viewed as saddle periodic orbits together with a funnel mechanism that puts trajectories onto its stable manifold (for details see [11]).

In order to further clarify the structure of state space, we numerically determined the basins of attraction of the three attractors displayed in Fig. 2(a) in two-dimensional sections of state space. The example shown in Fig. 2(b) reveals that attractors are surrounded by basins of attraction of other attractors as predicted by the above analysis. Because of this basin structure, noise induces repeated attractor switching among unstable attractors. Starting from the orbit defined by (3) the system may switch within sets of only six periodic orbit attractors as is apparent from the basins shown in Fig. 2(b). However, in larger networks [cf., e.g., Fig. 1(a)] a cluster can split up in a combinatorial number of ways, and exponentially many periodic orbit attractors are present among which the system may switch. The larger such networks are, the higher the flexibility they exhibit in visiting different attractors and exploring state space.

The preceding analysis demonstrates the existence of unstable attractors. To answer the question of how common unstable attractors actually are, we numerically estimated the fraction $p_{\mathrm{u}}(N)$ of state space occupied by basins of unstable attractors. As an example, Fig. 3 (inset) displays $p_{\mathrm{u}}(N)$ for $\varepsilon=0.2$ and $\tau=0.15$. While unstable attractors are absent if networks are too small (here $N \leq$ 4) and coexist with stable attractors in larger networks, the fraction $p_{\mathrm{u}}(N)$ approaches one for $N \gg 1$. More generally, we observed that $p_{\mathrm{u}}(N)$ approaches either zero or one in large networks, depending on the parameters. For networks of $N=100$ oscillators Fig. 3 shows the region of parameter space in which unstable attractors prevail $\left[p_{\mathrm{u}}(100)>0.5\right]$. As this region covers a substantial part of parameter space, precise parameter tuning is not needed to obtain unstable attractors. Furthermore, we find the same qualitative behavior independent of the detailed form of $U$. Hence, the occurrence of unstable attractors is a robust collective phenomenon in this model class of networks of excitatorily pulse-coupled oscillators.

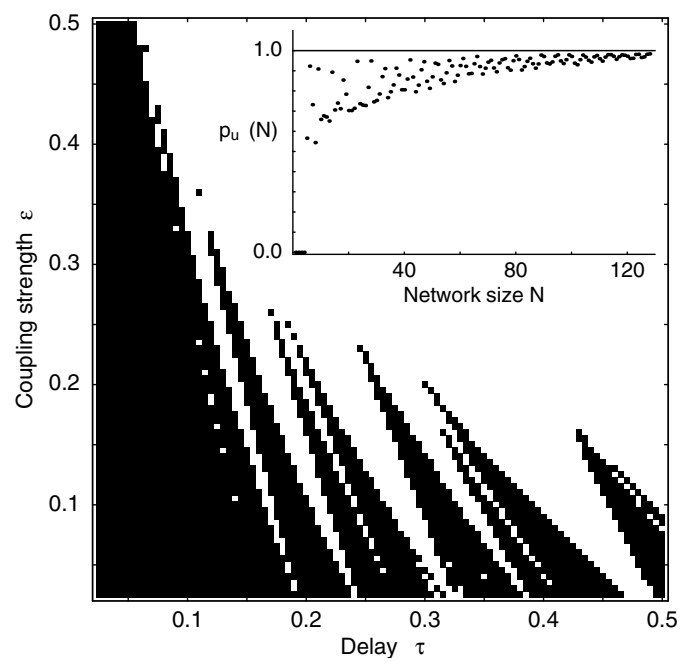

FIG. 3. Unstable attractors prevail for large networks and persist in a wide region of parameter space. Inset: $p_{\mathrm{u}}(N)$ for $N \leq 128, \varepsilon=0.2, \tau=0.15$. Main figure: Parameters with $p_{\mathrm{u}}(100)>0.5$ are marked in black. 
Unstable attractors persist under various classes of structural perturbations. For instance, preliminary studies on networks with randomly diluted connectivity suggest that a symmetric, all-to-all coupling is not required. Moreover, it is expected that every system obtained by a sufficiently small structural perturbation from the one considered here will exhibit a similar set of saddle periodic orbits, because linearly unstable states can generally not be stabilized by such a perturbation. Although, in general, these orbits may no longer be attracting, their dynamical consequences are expected to persist. In particular, a switching along heteroclinic connections may occur in the presence of noisy or deterministic, timevarying signals. As in the original system, the sequence of states reached may be determined by the directions into which such a signal guides the trajectory. By increasing and decreasing the strength of this signal, the time scale of switching may be decreased and increased, respectively, due to the linear instability. Interestingly, it has recently been shown that certain models of neural networks are capable of dynamically encoding information as trajectories near heteroclinic connections [14].

Furthermore, switching among unstable states also occurs in systems of continuously, phase-coupled oscillators $[15,16]$ that can be obtained from pulse-coupled oscillators in a certain limit of weak coupling [17]. In particular, Hansel, Mato, and Meunier show that a system of phase-coupled oscillators may switch back and forth among pairs of two-cluster states [15]. Working in the limit of infinitely fast response, i.e., discontinuous phase jumps, we have demonstrated that far more complicated switching transitions can occur in large networks if the oscillators are pulse-coupled.

In this Letter, we have presented the first example of a dynamical system, a network of pulse-coupled oscillators, that naturally exhibits attracting periodic orbits that are unstable. Intriguingly, these unstable attractors are located remote from the volume of their own basin of attraction. We have shown that they prevail in large networks and for a wide range of parameters. Whereas unstable periodic orbits are essential for the dynamics of many nonlinear systems, unstable attracting periodic orbits previously seemed to be exceptional cases. Our results indicate that in a class of systems of pulse-coupled units unstable attractors are the rule rather than the exception.

We thank A. Aertsen, M. Diesmann, U. Ernst, D. Hansel, K. Kaneko, K. Pawelzik, and C. v. Vreeswijk for useful discussions.

[1] R. E. Mirollo and S. H. Strogatz, SIAM J. Appl. Math. 50, 1645 (1990).

[2] U. Ernst, K. Pawelzik, and T. Geisel, Phys. Rev. Lett. 74, 1570 (1995); Phys. Rev. E 57, 2150 (1998).

[3] M. Tsodyks, I. Mitkov, and H. Sompolinsky, Phys. Rev. Lett. 71, 1280 (1993); C. van Vreeswijk, Phys. Rev. E 54,
5522 (1996); W. Gerstner, Phys. Rev. Lett. 76, 1755 (1996); W. Gerstner, J. L. van Hemmen, and J. D. Cowan, Neural Comput. 8, 1653 (1996); M. Diesmann, M.-O. Gewaltig, and A. Aertsen, Nature (London) 402, 529 (1999); P. C. Bressloff and S. Coombes, Neural Comput. 12, 91 (2000); S. H. Strogatz, Nature (London) 410, 268 (2001); D. Hansel and G. Mato, Phys. Rev. Lett. 86, 4175 (2001).

[4] J. P. Eckmann, Rev. Mod. Phys. 53, 643 (1981); J. Guckenheimer and P. Holmes, Nonlinear Oscillations, Dynamical Systems, and Bifurcations of Vector Fields (Springer, New York, 1983); A. Katok and B. Hasselblatt, Introduction to the Modern Theory of Dynamical Systems (Cambridge University Press, Cambridge, U.K., 1995).

[5] C. S. Peskin, Mathematical Aspects of Heart Physiology (Courant Institute of Mathematical Sciences, New York, 1984); J. Buck, Q. Rev. Biol. 63, 265 (1988); A. V. M. Herz and J. J. Hopfield, Phys. Rev. Lett. 75, 1222 (1995).

[6] J. Milnor, Commun. Math. Phys. 99, 177 (1985); see also J. Buescu, Exotic Attractors (Birkhäuser, Basel, 1997).

[7] J. C. Sommerer and E. Ott, Nature (London) 365, 138 (1993); K. Kaneko, Phys. Rev. Lett. 78, 2736 (1997); P. Ashwin and J. R. Terry, Physica (Amsterdam) 142D, 87 (2000); H. L. Yang, Phys. Rev. E 63, 036208 (2001).

[8] J. J. Hopfield, Proc. Natl. Acad. Sci. U.S.A. 79, 2554 (1982); Pulsed Neural Networks, edited by W. Maass and C. M. Bishop (MIT Press, Cambridge, 1999).

[9] We fix $\varepsilon=0.2, \tau=0.15$, and $U(\phi)=\ln \left[1+\left(e^{b}-\right.\right.$ 1) $\phi] / b$ with $b=3.0$. The orbits considered are structurally stable in a neighborhood of these parameters and the function $U$.

[10] Here $A=U^{-1}(U(\tau)+\hat{\varepsilon}), B=U^{-1}\left(U\left(1+2 \tau-a_{4}\right)+\right.$ $2 \hat{\varepsilon}), \quad C=C_{0}, \quad C_{\nu}=U^{-1}\left(U\left(U^{-1}\left(U\left(2 \tau+\nu U^{-1}(\hat{\varepsilon})\right)+\right.\right.\right.$ $\left.\left.\hat{\varepsilon})+1+\tau-a_{4}\right)+2 \hat{\varepsilon}\right)$ for $\nu \in\{0,1\}$ with recursively defined $a_{i}=U^{-1}\left(k_{i} \hat{\varepsilon}+U\left(\tau+a_{i-1}\right)\right)$ for $i \in\{1, \ldots, 4\}$, $a_{0}=0$, and $k_{1}=k_{3}=k_{4}=1, k_{2}=2$. For details see [11].

[11] M. Timme, F. Wolf, and T. Geisel, e-print cond-mat/ 0209432.

[12] Here $C^{\prime}=C_{1} \approx 0.756 \neq C=C_{0}$ because after a general perturbation $\boldsymbol{\delta}$ clusters are split up such that, in particular, $\phi_{1}<\phi_{2}$. Hence, oscillator 6 receives the later signal (from oscillator 1) only after it is reset by the earlier signal (from oscillator 2). Thus, the system jumps away from the original orbit and the orbit approached in the limit $\boldsymbol{\delta} \rightarrow \mathbf{0}$ is different from the original one. There exist, however, other unstable attracting periodic orbits that do not show this effect. For details see [11].

[13] $\boldsymbol{F}$ is defined by $F_{2}(\boldsymbol{\delta})=0, F_{3}(\boldsymbol{\delta})=\Delta L+H(\tau-\Delta L)-$ $A, \quad F_{4}(\boldsymbol{\delta})=H(\tau+\Delta L)-A, \quad F_{5}(\boldsymbol{\delta})=H(H(1+2 \tau-$ $\left.\left.L_{4}\right)+\Delta L\right)-B, \quad F_{6}(\boldsymbol{\delta})=H\left(\Delta L+H\left(\tau+1-L_{3}+\right.\right.$ $\left.\left.H\left(2 \tau-\delta_{2}+H\left(\delta_{2}\right)\right)\right)\right)-C^{\prime}$ where we abbreviate $H(\phi)=U^{-1}(U(\phi)+\hat{\varepsilon}), \quad L_{i} \equiv L_{i}(\boldsymbol{\delta})=H(\tau+H(\tau-$ $\left.\left.\delta_{2}+H\left(\delta_{2}+H\left(\tau+\delta_{i}-\delta_{2}+H(\tau)\right)\right)\right)\right)$ for $i \in\{3,4\}$ and $\Delta L=L_{4}-L_{3}$.

[14] M. Rabinovich et al., Phys. Rev. Lett. 87, 068102 (2001).

[15] D. Hansel, G. Mato, and C. Meunier, Phys. Rev. E 48, 3470 (1993).

[16] H. Kori and Y. Kuramoto, Phys. Rev. E 63, 046214 (2001).

[17] Y. Kuramoto, Physica (Amsterdam) 50D, 15 (1991). 